Epigenetische Mechanismen embryonaler Induktion und sozialer Prägungsprozesse

$\begin{aligned} \text { Authors: } & \\ \text { Submitted: } & 30 \\ \text { Published: } & 30 . \\ \text { Volume: } & 2 \\ \text { Issue: } & 5 \\ \text { Keywords: } & \text { Epid }\end{aligned}$

Horst Kress

30. August 2015

30. August 2015

2

5

Epigenetik, embryonale Induktion, soziale Prägung, Determination, epigenetischer Synapsencode, Gedächtnis;

Epigenetics, embryonal induction, social imprinting, determination, epigenetic synapse code, memory

DOI: $\quad$ 10.17160/josha.2.5.52 


\section{Epigenetische Mechanismen embryonaler Induktion und sozialer Prägungsprozesse}

Bei der Entwicklung eines vielzelligen Organismus aus dem befruchteten Ei erfolgt die Weitergabe genetischer Information durch identische Reduplikation der chromosomalen DNA und der sich anschließenden symmetrischen Weitergabe der Duplikate auf beide Tochterzellen im Verlauf sukzessiver mitotischer Teilungen. Auf diese Weise besitzen alle Körperzellen die gleiche genetische Information. Es drängt sich die Frage auf, wieso unter dieser Voraussetzung z. B. im menschlichen Körper mehr als 200 unterschiedliche Zelltypen entstehen können. Peter Spork schreibt in der Einleitung zu seinem Buch Der zweite Code (2009), dass dieser die Kernaussage der Epigenetik wiedergibt: „Der erste Code, die Buchstabenfolge der Gene, dominiert nicht alles. Es gibt noch ein weiteres biologisches Informationssystem. Ihm verdankt jede unserer Zellen, dass sie weiß, woher sie kommt, was sie erlebt und wohin sie geht." Die Grundlage für die Entstehung zellulärer Variabilität, d. h. von verschiedenen Differenzierungsmustern, stellen vorrangig induktive Mechanismen dar, die bei der strukturellen und funktionellen Integration von Zellverbänden im Verlauf der körperlichen Entwicklung am Werke sind. Induktive Prozesse basieren auf ein- oder gegenseitigen Signalwirkungen zwischen benachbarten Zellen, die auf die Expression von Genen Einfluss nehmen.

Es erscheint naheliegend, die Wirksamkeit derartiger Mechanismen nicht nur auf die frühembryonale und vorgeburtliche Entwicklung zu beschränken. Der Verhaltensforscher Konrad Lorenz (1903-1989) analogisierte erstmals bei Jungtieren Prozesse der Prägung von Verhaltensmustern mit Induktionsprozessen der Embryonalentwicklung. So schrieb er nach einem Hinweis auf den von dem Freiburger Embryologen Hans Spemann (1869-1941) näher definierten Begriff der embryonalen Induktion: „Es ist nicht sehr wesentlich, ob ein induzierender Einfluss von der Umgebung eines Gewebeanteils innerhalb eines Keimlings oder von der äußeren Umgebung eines Organismus ausgeht." (Lorenz 1973: 97). Doch ist ein derartiger Vergleich überhaupt zulässig? Zwei Aspekte sind dabei zu berücksichtigen: Erstens, liegen den Prozessen von Induktion und Prägung die gleichen materiellen Substrate, also die gleichen molekularen Komponenten zugrunde? Und zweitens sind die funktionellen Aspekte im Zellverband bzw. im Sozialverband miteinander vergleichbar? Dies würde Fragen u. a. zum Auftreten von Synergieeffekten durch Arbeitsteilung oder zur Vergleichbarkeit von körperlicher Homöostasie und sozialer Stabilität betreffen. Es gilt also im Folgenden, die Gültigkeit des von Lorenz geführten Vergleichs vor dem Hintergrund unseres aktuellen Wissens zu hinterfragen.

\section{Das Konzept der Induktion in der experimentellen Embryologie}

Wilhelm Roux (1850-1924) hatte mit seinem Programm zur „Entwicklungsmechanik“ die Embryologie um 1900 aus ihrer beschreibenden in die kausalanalytische Phase überführt (Roux, 1897). In seinem klassischen Experiment von 1887 an zweizelligen Froschkeimen tötete er eine der beiden Zellen mit einer heißen Nadel, trennte die Zellen aber nicht voneinander. Die überlebende Zelle entwickelte sich anschließend zu einem halbseitigen Embryo weiter, so als wäre der Keim aus einem Mosaik von zwei unabhängigen Hälften zusammengesetzt, also präformiert gewesen. Neben diesem Befund, der Roux' 
Prinzip der Selbstdifferenzierung entsprach, häuften sich in der Folgezeit allerdings nicht nur bei Amphibien immer mehr experimentelle Hinweise auf wechselseitige Abhängigkeiten zwischen den Teilen des Keims, die Roux als abhängige Differenzierung bezeichnete.

Der Schlüssel zum Prinzip der gegenseitigen Abhängigkeit, das für eine funktionelle Ganzeinheitlichkeit des sich entwickelnden Körpers sprach, wurde von Spemann entdeckt. Dieser hatte ein Instrumentarium entwickelt, mit dessen Hilfe er an Amphibienkeimen komplizierte Experimente durchführen konnte. Das richtungweisende Experiment seines Labors wurde von Hilde Pröscholdt (später H. Mangold) im Frühjahr 1921 an Molchkeimen durchgeführt (Mangold 1953: 166). Sie hatte bei einer Art mit schwacher Pigmentierung der Zellen (Triturus cristatus) aus einer als obere Urmundlippe bezeichneten Keimregion einen kleinen Bezirk entnommen und in den frühen Keim einer dunkel pigmentierten Art (T. taenatus) transplantiert. Das Ergebnis war überraschend: Im Wirt entstand zusätzlich zu seinem eigenen Kopf auf der gegenüberliegenden Seite ein zweiter, der allerdings nicht vom hellen Implantat stammte, sondern vom dunklen Wirt! Als Spemann diese Chimäre zum ersten Mal sah, erkannte er deren Bedeutung sofort: Das Implantat hatte im Wirt eine Entwicklungsleistung ausgelöst, die zur Festlegung einer zweiten Körperlängsachse führte. Er prägte für diese auslösende Region den Begriff Organisator. Ein Organisator induziert im umgebenden Zellgewebe, dessen Entwicklungspotenziale anfänglich noch offen und vielfältig sind (Totipotenz), die Festlegung einer einzigen Entwicklungsrichtung, die zu einem terminal determinierten Zustand und damit zu einem unipotenten Entwicklungsschicksal führt.

Die materielle Grundlage für Induktions- und Determinationsprozesse blieb für viele Jahrzehnte ungeklärt. Viele namhafte Forscher bissen sich an diesem Problem die Zähne aus. Für die Zeit zwischen 1930 und 1980 konstatiert der Entwicklungsbiologe Scott F. Gilbert (2001: 155): „the <primary induction problem> was considered a graveyard of biologists, a problem so fraught with non-specifity, uninterpretable results, and conflicting data, that a young biologist would be foolish to enter the morass". Die Versuche des Berliner Biochemikers Heinz Tiedemann (1923-2004), induzierende Faktoren mit Mitteln der konventionellen Biochemie bis zur Reinheit anzureichern, führten zwischen 1950 und 1980 zwar zu Teilerfolgen, wurden aber durch die wesentlich schnelleren und effektiveren Methoden der aufkommenden Molekularbiologie letztendlich überrollt (Slack 1999: 93).

\section{Entwicklungsgene und ihre funktionelle Modulation}

Die Frage nach den materiellen Substraten der Steuerung von Entwicklungsprozessen konnte erst durch die sich nach 1960 etablierende Entwicklungsgenetik beantwortet werden. Hier sind der englische Entwicklungsbiologe Conrad H. Waddington (1905-1975) und der schweizerische Zoologe und Entwicklungsgenetiker Ernst Hadorn (1902-1976) zu nennen. Beide hatten als klassische Zoologen begonnen, waren aber auf das von dem amerikanischen Zoologen und Genetiker Thomas H. Morgan (1866-1945) um 1910 in die genetische Forschung eingeführte Modell der Fruchtfliege Drosophila melanogaster umgestiegen. Waddington wurde bekannt durch seine Metapher der epigenetischen Landschaft, mit der er die Beziehungen zwischen dem Genotyp und dem Phänotyp als sich zunehmend 
spezialisierende Entwicklungsbahnen darzustellen versuchte. Er prägte den Terminus Epigenetik, der sich von der Fusion der Begriffe Epigenesis und Genetik ableitet. Allerdings blieben Waddington's eigene wissenschaftliche Arbeiten ohne größere Nachhaltigkeit. Ganz im Gegensatz zu Hadorn, dessen Entdeckung der Transdetermination (Änderung des determinierten Zustands) und des Postulats des zeitund organspezifischen stufenweisen Einsatzes von Genen während der Individualentwicklung (Hadorn, 1955) der Entwicklungsgenetik nicht nur in Europa wesentlichen Auftrieb gab. Zu seinen bekanntesten Schülern zählt Walter Gehring (1939-2014), in dessen Labor 1975 die Tübinger Molekularbiologin Christiane Nüsslein-Volhard (1942-) und der aus den USA stammende Eric Wieschaus (1947-) eine jahrelange Zusammenarbeit begründeten. Für ihre Arbeiten zur genetischen Kontrolle der frühen Embryonalentwicklung von Drosophila am European Molecular Biology Laboratory (EMBL) in Heidelberg wurde ihnen 1995, zusammen mit dem amerikanischen Entwicklungsgenetiker Ed Lewis (1918-2004) der Nobelpreis für Medizin verliehen.

Diese Arbeiten legten den Grundstein für die genetische und molekularbiologische Analyse von Entwicklungsgenen zunächst bei Drosophila, später dann aber auch bei einer Vielzahl anderer tierischer Modelle. Hierbei wurde die faszinierende Entdeckung gemacht, dass diese Gene im gesamten Tierreich in Aufbau und Funktion eine ungeahnte Einheitlichkeit aufweisen. Ab den frühen 1970er Jahren, beginnend mit der erstmaligen Klonierung eines Entwicklungsgens von Drosophila durch den amerikanischen Molekularbiologen David Hogness (1925-), konnte man bei Eukaryonten Gene und deren Produkte durch Amplifikationsmethoden in Mengen gewinnen, die strukturelle und funktionelle Analysen möglich machten. Es zeigte sich, dass die große Mehrheit von Entwicklungsgenen für zwei verschiedene Proteingruppen kodiert. Die erste Gruppe umfasst die Transkriptionsfaktoren, die mit Schalterelementen von Genen interagieren und auf diese Weise deren Aktivität im Kern steuern. Bei der anderen Gruppe handelt es sich um diffusionsfähige Signalproteine, die von Zellen abgegeben werden und an spezifischen Rezeptoren in den Membranen benachbarter Zellen andocken. In diesen werden Signalkaskaden ausgelöst, die im Zytoplasma und im Kern zu spezifischen Reaktionen führen. Entwicklungsprozesse entpuppten sich als funktionelle Wirkungsketten von Transkriptionsfaktoren und Signalproteinen, die in räumlich und zeitlich genau festgelegten interaktiven Netzwerken einem Endzustand entgegenstreben, der letztendlich den entwicklungsphysiologisch determinierten Zustand einer Zelle im Sinne Spemanns repräsentiert.

Dieser Zustand muss allerdings über die gesamte Lebensdauer einer differenzierten Zelle aufrechterhalten werden. Dazu sind Mechanismen erforderlich, die anfänglich labil eingestellte Regelgrößen dauerhaft fixieren. Die Lösung dieses Problems geht ebenfalls auf frühe Beobachtungen bei Drosophila zurück. 1930 hatte Hermann J. Muller (1890-1967), der Entdecker der mutagenen Wirkung von Röntgenstrahlung, erstmalig eine Drosophila-Mutante beschrieben, bei der die rote Färbung der Komplexaugen verändert ist. Anstatt der normalerweise einheitlichen Rotfärbung aller Ommatidien weisen die Augen dieser Mutante eine rot-weiß-Scheckung auf. Das für die Rotfärbung verantwortliche Gen $\left(\right.$ white $\left.{ }^{+}\right)$ist in weißen Ommatidien in seiner Funktion unterdrückt. Die zytologische Analyse der Chromosomen ergab, dass es sich um eine Mutation (Inversion) des X-Chromosoms handelt, bei der das white ${ }^{+}-$Gen in die Nähe von Heterochromatin verlagert ist. Dieses ist, wie auch das Euchromatin, eine strukturgebende Komponente der Chromosomen. Das Heterochromatin ist durch eine spezielle 
Basenabfolge der DNA und der Zusammensetzung chromosomaler Proteine gekennzeichnet, die in ihrem Einflussbereich zur Repression der Genaktivität führt.

Dieser als position-effect-variegation (PEV) bezeichnete Effekt war somit der erste Hinweis darauf, dass die Aktivität von Genen vom Aufbau des umgebenden Chromatins abhängig ist. Im Laufe der Zeit häuften sich die Indizien für eine derartige Abhängigkeit in den verschiedensten eukaryontischen Zelltypen, angefangen bei Hefen, über Pflanzen und Tiere bis hin zum Menschen. Man entdeckte eine kaum überschaubare Vielfalt von Mechanismen, die Chromatin in einem offenen bzw. in einem geschlossenen Zustand halten. Die Grundlage dafür stellen neben vielen anderen Proteinen, vor allem Histone dar, die als basische, $d$. h. positiv geladene Moleküle mit der negativ geladenen DNA interagieren und mit ihr Molekülkomplexe bilden. Diese Komplexbildung kann durch enzymatische Modifikationen von Struktur und Ladung (z. B. durch Acetylierung, Methylierung oder Phosphorylierung) variiert werden. Wir sprechen in diesem Zusammenhang von Chromatinschreibern. Durch sie entsteht eine ungeahnte Variationsbreite der Topographie von Chromatinstrukturen, die wiederum durch Chromatinleser als für Transkriptionsfaktoren offenes oder geschlossenes Chromatin interpretiert werden. Entscheidend ist, dass bei diesen Vorgängen die angeborene Basenabfolge der DNA nicht verändert wird. Es handelt sich also nicht um echte Mutationen, sondern lediglich um strukturelle Modifikationen des Chromatins, die von Zelle zu Zelle, unter Umständen auch von Generation zu Generation weitergegeben werden. Wir sprechen bei der Gesamtheit all dieser Mechanismen vom Histoncode des Chromatins. Entscheidend ist, dass in der Individualentwicklung die Festlegung des Histoncodes einer jeden Körperzelle von inneren und äußeren Umständen abhängig ist, also erworben wird.

Dazu kommt noch ein zweiter Mechanismus, nämlich die bereits bei Bakterien anzutreffende DNA-Methylierung. Sie stellt ebenfalls keine sequenzverändernde Mutation dar, sondern eine chemische Modifikation der Base Cytosin. Diese beeinflusst aus sterischen Gründen die Interaktion von Transkriptionsfaktoren oder anderen Proteinen mit der DNA. Insgesamt werden die Veränderungen des Histoncodes und der Muster methylierter DNA als epigenetischer Code bezeichnet, allerdings nicht im ursprünglichen Sinne einer zeitlich und räumlich strukturierten Kausalkette, sondern im Sinne einer hierarchischen Pyramide, in der die Gene funktionell wirksamen Überstrukturen untergeordnet sind. Letztere sind ihrerseits von räumlichen und zeitlichen Einflüssen abhängig. Sie ermöglichen eine Anpassung genetischer Expressionsmuster (in ihrer Gesamtheit als Transkriptom bezeichnet) des sich entwickelnden Organismus an sowohl konstante als auch an variable Umweltbedingungen (vgl. dazu auch den Beitrag von Barbara Tzschentke in diesem Band). Diese phänotypische Flexibilität, die 1909 von dem deutschen Zoologen R. Woltereck (1877-1944) als Reaktionsnorm bezeichnet wurde, gewährleistet einem Organismus während der Individualentwicklung durch physiologische Anpassungsfähigkeit größtmögliche Überlebens- und Fortpflanzungschancen. 


\section{Prägung als entwicklungsabhängige Kanalisierung neuronaler Verknüpfungen}

Spielen sich nun, wie der Lorenzsche Funktionsvergleich von Embryonalentwicklung und Verhalten nahelegt, bei der Prägung als spezieller Form des Lernens ähnliche molekulare Vorgänge ab wie beim embryonalen Determinationsgeschehen? Um sich einer Beantwortung dieser Frage anzunähern, bedarf es eines kurzen Rückblicks auf verhaltensgenetische Befunde aus den 1960er Jahren. Damals begann der zur Entwicklungsbiologie konvertierte Physiker Seymor Benzer (1921-2007) am Caltech in Pasadena genetische Mutanten von Drosophila zu isolieren, die in ihrem Verhalten auffällige Veränderungen zeigten. ${ }^{1}$ Von den vielen Mutanten, die Benzer und seine Schüler im Laufe der Zeit isolierten, seien an dieser Stelle nur zwei genannt, nämlich rutabaga und dunce. Beide Mutanten zeigen schwache Leistungen im Lernen und vergessen das Gelernte auch sehr schnell, $d$. h. sowohl die Bildung als auch die Aufrechterhaltung von Gedächtnisinhalten sind signifikant beeinträchtigt. Spätere Analysen in anderen Labors ergaben, dass es sich bei den von diesen Genen codierten Proteinen um Enzyme handelt, die an der Synthese und dem Abbau des cyclischen Adenosinmono-phosphats (cAMP) beteiligt sind. Für dieses Molekül war etwa zur gleichen Zeit durch die Arbeiten an der Meeresschnecke Aplysia californica im Labor des amerikanischen Neurobiologen Eric Kandel (1929-) an der Columbia University in New York gezeigt worden, dass es bei der Gedächtnisbildung eine zentrale Rolle spielt. ${ }^{2}$

Es existieren zwei Formen der Gedächtnisbildung, nämlich das Kurzzeitgedächtnis und das Langzeitgedächtnis. Beim Kurzzeitgedächtnis, das sich bei relativ kurzfristigen Reizen oder Eindrücken bildet, vermittelt CAMP durch Aktivierung bestimmter zytoplasmatischer Signalketten lediglich die Stärkung der in den Neuronen bereits existierenden Synapsen. Bei längeren und/oder intensiveren Reizen hingegen, verursacht CAMP im Kern die Aktivierung des Transkriptionsfaktors CREB (cAMP Response Element Binding-Protein). Die dadurch bedingte Veränderung des Transkriptoms und die damit verbundene Synthese neuer Proteine führt neben der Stärkung der bereits vorhandenen Synapsen zusätzlich zur Bildung neuer Synapsen und schließlich zum Aufbau eines mehr oder minder dauerhaften Langzeitgedächtnisses. Entscheidend ist also, dass - im Gegensatz zum Kurzzeitgedächtnis die Bildung des Langzeitgedächtnisses von der Proteinneusynthese abhängig ist.

Im Kontext früher Prägungsprozesse ist die Gedächtnisbildung allerdings durch zwei Besonderheiten gekennzeichnet: 1) die Beschränkung auf ein peri- oder postnatales Zeitfenster (sensible Phase), das unter Umständen nur wenige Stunden offen ist und 2) deren intensive, oft lebenslange Wirkung. Sie unterscheiden sich dadurch von späteren Lern- und Prägungsprozessen in der Juvenilund/oder Adultphase, die sich über längere Zeiträume erstrecken und dem mehr oder minder schnellen Vergessen unterliegen können. Konrad Lorenz zeigte eindrucksvoll, dass die Nachlaufprägung bei Graugänsen und anderen Vögeln ein angeborenes Verhaltensschema darstellt, das nicht auf das arteigene Objekt (im Normalfall die eigene Mutter) fixiert ist (Lorenz 1935). Jedes Objekt, das sich nach

\footnotetext{
${ }^{1}$ Seine Biographie ist von J. Weiner (2002), eingebettet in die spannende frühe Geschichte der Genetik, überaus eindrucksvoll dargestellt.

${ }^{2}$ Auf die Umstände dieser Entdeckung näher einzugehen, würde hier zu weit führen. Sie sind in Kandels Autobiographie Auf der Suche nach dem Gedächtnis (Kandel 2007) im Kontext der Entwicklung der modernen Neurobiologie allgemein verständlich beschrieben.
} 
dem Schlüpfen in der Nähe des Kükens bewegt und Töne von sich gibt, löst die dauerhafte Instinkthandlung des Nachlaufens aus, die eine Vorbedingung für dessen Sozialisierung ist. Man könnte diese Situation am besten mit einer gespannten Mausefalle vergleichen, die unabhängig vom auslösenden Objekt bei Berührung zuschlägt. Diese lebenswichtige Bereitschaft zum Prägungsverhalten setzt voraus, dass „ein ganz bestimmter physiologischer Entwicklungszustand des Jungtieres“ (Lorenz 1935:142) vorliegt. Dieser Zustand, den wir für alle Prägungsprozesse, sei es mit unterschiedlicher Dauer oder auch mit unterschiedlichem zeitlichen Auftreten, annehmen müssen, hängt in erster Linie mit der nach dem Schlüpfen/der Geburt noch nicht abgeschlossenen Gehirnentwicklung zusammen.

Bei vielen Wirbeltieren geht der Eintritt in die Sozialisierungsphase u. a. mit dem Öffnen der Augen und mit der damit verbundenen frühen postnatalen Entwicklung des visuellen Systems einher. Bei den zu den Primaten gehörenden Marmosets etwa erstreckt sich der vollständige Aufbau der verschiedenen Schichten des primären visuellen Kortex nach der Geburt, in Abhängigkeit von der Reifung der Retina, über einen Zeitraum von rund zwei Jahren (Bourne et al., 2005). Beim Menschen schätzt man, dass die Synapsendichte im präfrontalen Kortex (eine zentrale Integrationsstelle von sensorischen Eingängen und Gedächtnisinhalten) in den ersten zwei bis drei Lebensjahren um etwa das Doppelte zunimmt (Abb. 13.1, Phase 3). Dies beruht vornehmlich auf dem Ausbau des neuronalen Vernetzungsgrades von Axonen und Dendriten (graue Zellmasse). Bourgeois (2010: 73) schätzt, dass im gesamten cerebralen Kortex des menschlichen Babys im perinatalen Zeitraum jede Sekunde (!) hunderte von Millionen, wenn nicht sogar Milliarden neuer Synapsen gebildet werden. Es ist dies der erste, mit einer sensiblen Periode vergleichbare Lebensabschnitt, in dem beim Menschen am Objekt der Mutter die Fähigkeit zur sozialen Bindung aufgebaut wird (Mahler et al., 1997). Bei der Strauchratte Octodon degus wurde beobachtet, dass bei Störung einer derartigen Entwicklung durch Elterndeprivation sowohl im präfrontalen Kortex als auch in anderen Gehirnarealen, die Synapsendichte signifikant von der Norm abweicht (Bock et al., 2003). Dies kann eine Grundlage für spätere pathologische Verhaltensweisen (z. B. verstärkte Angstgefühle) darstellen. Es besteht also eine zeitliche Korrelation zwischen postnatalen Prägungsprozessen und der fortschreitenden Differenzierung des jugendlichen Gehirns. Dies kann dahingehend interpretiert werden, dass junge, noch unausgereifte Neuronen in der Lage sind, in relativ kurzer Zeit starke und dauerhafte synaptische Kontakte zu bilden. Man geht inzwischen davon aus, dass im jugendlichen Großhirn ein zunächst noch nicht gefestigtes neuronales Netzwerk vorliegt. Auf diesem plastischen Substrat werden jene Vernetzungen als stabil aufgebaut, die durch die persönliche Lebensgeschichte epigenetisch festgelegt werden. Dieser Lebensabschnitt, in dem sich die Synapsendichte in einer Plateauphase (Abb. 13.1, Phase 4) befindet, dauert beim Menschen etwa eine Dekade bis zum Beginn der Pubertät. Es ist dies die Phase höchster Lernkapazität. Während der Pubertät werden bis dahin nicht in Anspruch genommene Verknüpfungen wieder abgebaut. Der amerikanische Neurobiologie Donald Hebb (1904-1985) hatte ein derartiges Verhalten bereits 1949 beschrieben (Hebb'sche Regel). Dieser Vorstellung entspricht die Schätzung, dass im menschlichen präfrontalen Kortex im Verlauf der Pubertät die Synapsendichte wieder um etwa $30 \%$ abnimmt (Abb. 13.1). Diesem schnellen Abbau folgt während der Adultphase ein langsamerer Abbau, der sich im Verlauf der Seneszenz wieder beschleunigt (Abb.13.1, Phase 5). Wir haben es also mit einer entwicklungsabhängigen Kanalisierung neuronaler Verknüpfungen, d. h. einer Einschränkung der 
neuronalen Flexibilität und Plastizität und damit auch der Lernfähigkeit zu tun. Dies ist vergleichbar mit dem Verlust der Pluripotenz embryonaler Körperzellen im Verlauf von Determinationsprozessen.

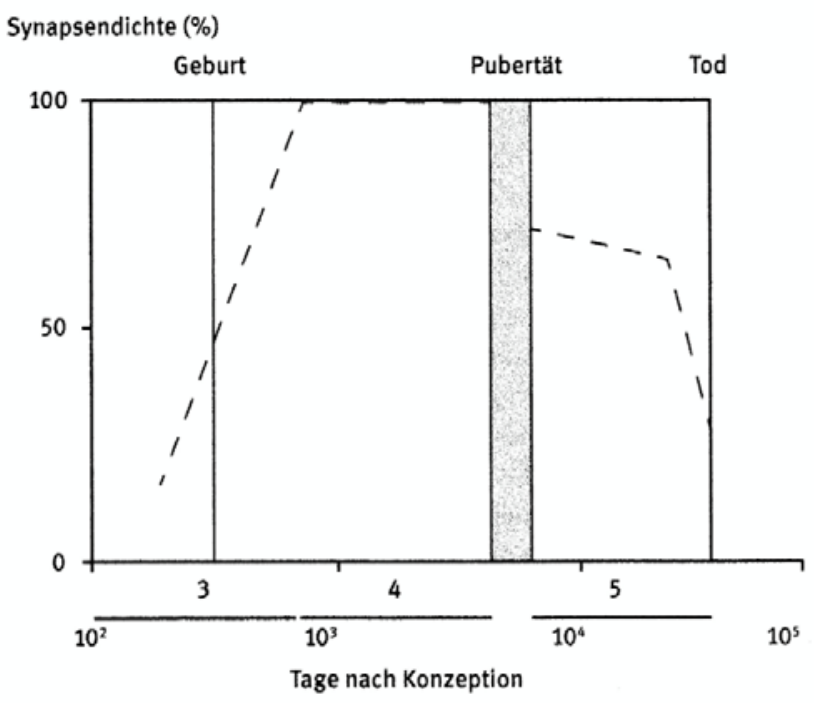

Abb. 13.1: Veränderungen der Synapsendichte des präfrontalen Kortex im Verlauf des menschlichen Lebens. Ordinate: Synapsendichte (gestrichelte Linie) bezogen auf den maximalen Wert (in Prozent). Abszisse: Alter in Tagen nach Empfängnis (logarithmische Skala). Phasen der Synaptogenese (nummerierte Balken): Phase 3: genetisch festgelegtes schnelles Synapsenwachstum im peri-/postnatalen Zeitraum. Phase 4: Plateauphase im Juvenilstadium. Es ist dies eine „Dachkurve“, welche die Synaptogenese im Verlauf verschiedener Prägungs- und Lernphasen aufsummiert. Phase 5: Abnahme der Synapsendichte nach der Pubertät durch Einschränkung der Synaptogenese auf bestimmte Bereiche des Kortex. Modifiziert aus Bourgeois, 2010, Fig. 5.3., mit Genehmigung des Autors und von Cambridge University Press.

\section{Der epigenetische Synapsencode}

Doch liegen beiden Prozessen die gleichen molekularen Mechanismen zugrunde? Die embryonalen Induktionsprozesse involvieren interzelluläre Signalproteine und intrazelluläre Signalkaskaden, die im Kern zellspezifische Genexpressionsmuster etablieren. Diese werden anschließend durch den epigenetischen Code fixiert. Bei Prägung und Gedächtnisbildung laufen prinzipiell vergleichbare Prozesse ab. Allerdings sind bislang nur wenige Transkriptionsfaktoren identifiziert worden, die an der Gedächtnisbildung beteiligt sind. Zusätzlich zum bereits erwähnten CREB-Faktor ist hier vornehmlich cFos zu nennen, der z. B. bei der Sexualprägung männlicher Zebrafinken in den Zellkernen des Hippocampus um den Faktor 15 verstärkt produziert wird (Sadananda und Bischof, 2004). Das c-FosProtein ist Bestandteil des AP1-Faktors (Aktivator Protein 1), der generell am Regulationsgeschehen bei einer Vielzahl tierischer Entwicklungsprozesse beteiligt ist (Karin et al., 1997). 
Auch im Hinblick auf intrazelluläre Signalkaskaden unterscheiden sich Neuronen von anderen Körperzellen nur wenig. Gleiches gilt für die interzelluläre Kommunikation, wobei mit den Neurotransmittern, z. B. von Glutamat, Serotonin, Octopamin oder Dopamin eine für das Nervensystem qualitativ spezifische Komponente dazu kommt. Auch für die Frage nach der Fixierung von epigenetischen Codes deuten experimentelle Daten zunehmend auf einen Zusammenhang zwischen Prägungsprozessen und Veränderungen von DNA-Methylierungsmustern und von Histoncodes (Roth und Sweatt, 2009; Sweatt, 2009) hin. Prozesse der embryonalen Determination und der Gedächtnisbildung beruhen somit hinsichtlich der Bildung epigenetischer Codes im Zellkern auf vergleichbaren molekularen Mechanismen.

Das Problem ist aber vielschichtiger. Wie oben dargestellt, werden im Verlauf der postnatalen Entwicklung neuronale Netzwerke des Gehirns erfahrungsbedingt kanalisiert, wobei bereits existierende Synapsen, je nach Beanspruchung, entweder verstärkt oder zurückgebildet werden. Zusätzlich entstehen beim Aufbau eines Langzeitgedächtnisses neue Synapsen durch Proteinsynthese, die ihrerseits die Beteiligung des Kerns in Form bestimmter Genexpressionsmuster voraussetzt. Diese Mitwirkung ist aber keine conditio sine qua non. Frey und Morris (1997) konnten am Hippocampus des Rattengehirns zeigen, dass kurzfristig aktivierte Synapsen auch dann ein Langzeitgedächtnis ausbilden können, wenn die Proteinsynthese durch einen Inhibitor blockiert ist. Zwingende Voraussetzung dafür ist allerdings, dass kurz vorher andere Synapsen des gleichen Neuronenverbandes ein Langzeitgedächtnis aufgebaut haben. Offenbar sind Synapsen unabhängig vom Kern in der Lage, miteinander zu kommunizieren. In der Folgezeit entstand auf der Basis dieses und weiterer Befunde die synaptic tagging and capture hypothesis (STC-Hypothese, Redondo und Morris, 2011). Sie besagt, dass in den für die Bildung eines Langzeitgedächtnisses aktivierten Synapsen zunächst Veränderungen erfolgen, die als eine Art Markierung (tag) fungieren und zur strukturellen und funktionellen Stärkung eines Synapse führen. Es entstehen dabei auch plasticity related products (PRPs), die sich im Zytoplasma einer Nervenzelle verteilen und von anderen, schwach aktivierten, bereits mit einem tag versehenen Synapsen zum Aufbau eines neuen Langzeitgedächtnisses aufgegriffen werden können. Die Zusammensetzung der PRPs und die Art und Weise ihres Transports liegen noch im Bereich der Spekulation. Favorisiert werden u. a. mRNAs, die in Form von Ribonukleoprotein-Partikeln (RNPs) intrazellulär transportiert werden. In aktivierten Synapsen werden diese dann selektiv aufgenommen und die anschließend freigesetzten mRNAs an Ort und Stelle in Proteine, die zum Aufbau des Langzeitgedächtnisses in diesen Synapsen benötigt werden, translatiert. Es sind dies in erster Linie Komponenten des lokalen Zytoskeletts, sowie der prä- und postsynaptischen Membranen, insbesondere solche der Rezeptoren von Neurotransmittern. Für die ständige Versorgung von markierten Synpasen mit zytoplasmatischen PRPs wurde von Doyle und Kiebler (2011) das sushi belt-Modell vorgeschlagen. In Analogie zu einem ständig im Kreis laufenden Förder-band, von dem sich in japanischen SushiRestaurants die Gäste ihre Happen auf den Teller laden können, postulieren die Autoren in Neuronen die Existenz eines ständig in Bewegung befindlichen, aus Komponenten des Zytoskeletts aufgebauten Transportsystems, aus dem die „hungrigen“ Synapsen ihren Bedarf an PRPs decken können.

Die Komplexität dieser Vorgänge erhält allerdings durch Befunde in jüngster Zeit völlig neue Dimensionen. Vieles spricht nämlich dafür, dass neben der Kontrolle der Transkriptionsrate sowohl die 
Lebensdauer als auch die Translatierbarkeit von mRNAs in Nervenzellen durch eine Vielzahl verschiedener Mikro-RNAs moduliert wird (beim Menschen an die Tausend; Bredy et al., 2011: 89). Es handelt sich dabei um sehr kleine RNA-Moleküle, die mit mRNAs hybridisieren können und dadurch deren Funktionalität nicht nur im Kern, sondern auch in Dendriten und Synapsen nachhaltig beeinflussen. Dies wird nicht nur für die embryonale Gehirnentwicklung angenommen, sondern auch für Prozesse der neuronalen Plastizität und der Gedächtnisbildung im adulten Gehirn (Bredy et al., 2011: 91).

Insgesamt gesehen, impliziert die STC-Hypothese ein starkes Eigenleben von Synapsen. Sie reagieren auf kurzfristige Reize mit einer lokalen, zeitlich begrenzten Verstärkung ihrer Funktion, die wir als Kurzzeitgedächtnis kennen. Bei länger andauernden Reizen soll es zusätzlich zur Akkumulation von PRPs aus dem Zytoplasma kommen, die dann auch anderen Synapsen zur Bildung dauerhafter neuronaler Verknüpfungen zur Verfügung stehen. Es entsteht dadurch in einzelnen Neuronen oder auch in neuronalen Netzen ein Verstärkungseffekt, der zu einem spezifisches Muster von langfristig aktivierten Synapsen führt, die einen bestimmten dauerhaften Gedächtnisinhalt repräsentieren. Ich möchte an dieser Stelle dieses Muster - in Analogie zum epigenetischen Code des Kerns - als epigenetischen Synapsencode bezeichnen. Für beide Codes ist eine gegenseitige Abhängigkeit zu erwarten: In jugendlichen und/oder frisch geborenen Neuronen dürfte der die anfängliche Entwicklung steuernde neuronale Kerncode zunächst das gesamte Potenzial von Reaktionen neuronaler Strukturen auf Umwelteinflüsse, z. B. in sensiblen Perioden ermöglichen. Die Art und Weise dieser Einflüsse würde anschließend selektiv den sich davon ableitenden, prägungs- und lernspezifischen Synapsencode etablieren. Aus meiner Sicht dürfte es für dessen langfristige Aufrechterhaltung notwendig sein, über eine Rückkoppelung mit dem Kern dessen epigenetische Codierung dem Synapsencode anzupassen. Der Kerncode würde somit, entsprechend einem embryonalen Determinationsvorgang, von der neuronalen Pluripotenz in eine Unipotenz kanalisiert, die mit dem Synapsencode eine interaktive, funktionelle Einheit bildet. Es entspräche dies der Konsolidierung des Langzeitgedächtnisses.

\section{Formale Identität zwischen embryonaler Induktion und sozialer Prägung}

Bei geschlechtlicher Fortpflanzung entsteht während der Embryonalentwicklung durch Teilungsvorgänge aus dem befruchteten Ei ein ganzheitlicher Zellverband, den wir als Organismus bezeichnen. Dieser hat zwei Aufgaben: Erstens, sein individuelles Leben aufrechtzuerhalten und zweitens den Fortbestand der Art durch erfolgreiche Fortpflanzung zu sichern. Beide Aufgaben werden im Zellverband durch Arbeitsteilung erzielt. Die dafür notwendigen Strukturen und Organe werden durch Vermittlung chemischer Signale in Differenzierungsprozessen gebildet und deren Funktionen homöostatisch miteinander integriert. Der Ablauf dieser Prozesse wird in erster Linie durch die genetische Codierung bestimmt (Abb. 13.2). Diese legt zeitlich und räumlich fest, wann welche Faktoren an welcher Stelle ins Spiel kommen (Selbstdifferenzierung). Dieses Programm ist angeboren. Der Ablauf dieser Interaktionen wird sekundär von der inneren und äußeren Umwelt eines Zellverbands moduliert (abhängige Differenzierung). Die epigenetische Modellierung ermöglicht die optimale Anpassung des Organismus an 
seine gegebene Umwelt. Die Fixierung der erworbenen Transkriptome erfolgt durch spezielle Muster der DNA-Methylierung und durch Histoncodes, die zusammen den epigenetischen Kerncode bilden.

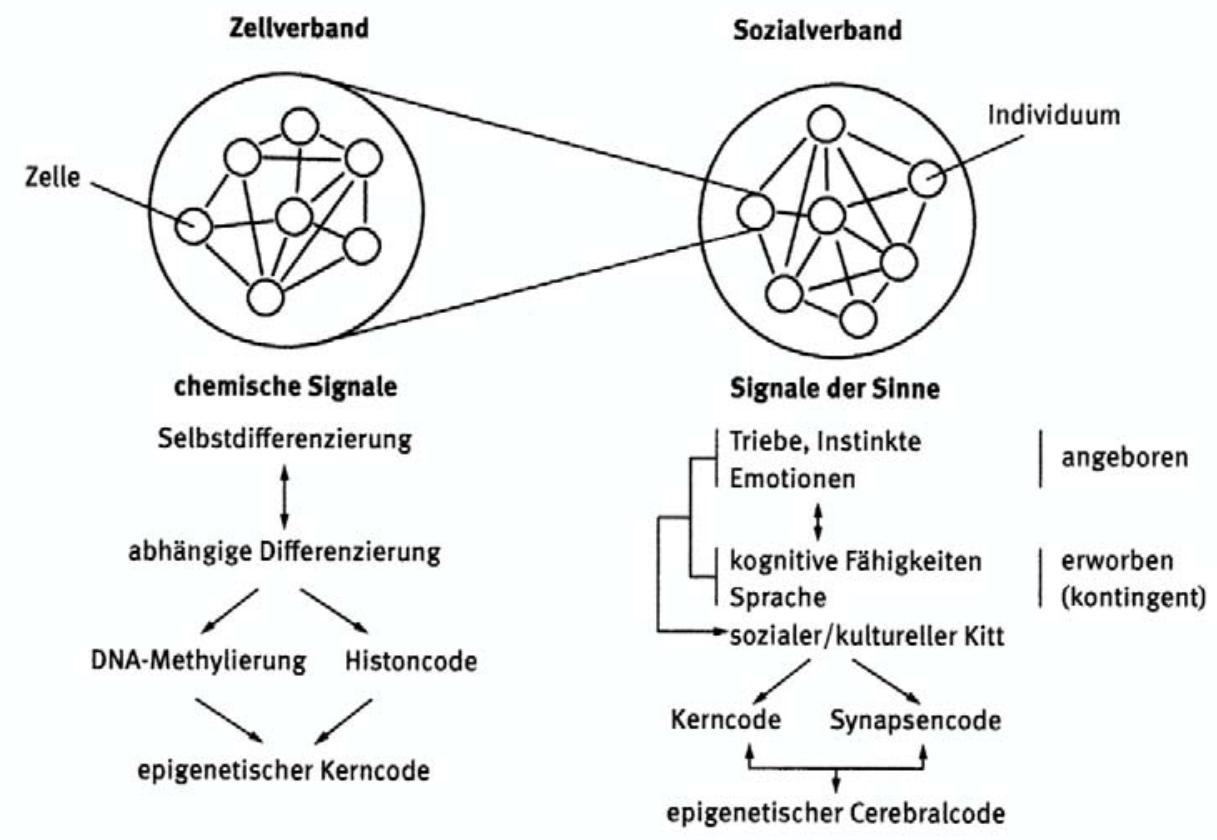

Abb. 13.2: Vergleichende Darstellung des funktionellen Ablaufs von Determinationsprozessen in der Embryonalentwicklung eines Individuums und seiner Eingliederung in einen Sozialverband durch Prägungs- und Lernprozesse. Nähere Einzelheiten siehe Text.

Bei sozialen Prägungsprozessen rückt an die Stelle des Zellverbands der aus Individuen bestehende Sozialverband. Seine Aufgabe ist es, erstens das Überleben der einzelnen Individuen bis in die Fortpflanzungsphase zu sichern und damit zweitens den Fortbestand der Population zu gewährleisten. Beide Funktionen werden - wie im Zellverband - ebenfalls durch Arbeitsteilung erzielt. Die Kommunikation erfolgt über Signale der Sinne, also z. B. akkustisch, optisch oder olfaktorisch. Die Interpretation dieser Signale spielt sich interaktiv auf zwei Ebenen ab. Es ist dies zunächst die angeborene Ebene der Triebe, Instinkte und Emotionen, die bei Vertebraten primär im Stammhirn lokalisiert sind (limbisches System). Wir können dies mit der genetisch fixierten Selbstdifferenzierung im Zellverband vergleichen. Diese Ebene wird ergänzt bzw. überlagert durch die Ebene des Großhirns, das sich im Verlauf der Evolution aus dem dorsalen Bereich des Riechhirns (Telencephalon) gebildet und bei Säugern, insbesondere aber beim Menschen außerordentliche Dimensionen und Fähigkeiten entwickelt hat. 
Die morphologische Entwicklung des Großhirns und das Potenzial seiner mentalen Leistungen sind genotypisch festgelegt. Es steht außer Zweifel, dass angeborene Prädispositionen neuronaler Verknüpfungen existieren, die wir als Veranlagung oder als Talent bezeichnen. Die endgültige Funktionalität des Großhirns wird allerdings erst im Verlauf seiner Entwicklung durch Prägungs- und Lernprozesse erworben. Dies ist vergleichbar mit der abhängigen Differenzierung in der Embryonalentwicklung. Die im Rahmen von Kultur und Sprache erworbenen kognitiven Fähigkeiten des Individuums schaffen in Verbindung mit der rationalen Kontrolle der angeborenen Funktionen des limbischen Systems die homöostatisch wirkenden Funktionen des Sozialen und Kulturellen, die für das Wohl des Einzelnen und für die Beständigkeit eines Sozialverbands zwingend erforderlich sind. Wie in der Embryonalentwicklung erfolgt die Fixierung der Transkriptome des individuellen Gehirns zunächst durch epigenetische Kerncodes. Dazu kommen aber nun die zusätzlichen und entscheidenden, ebenfalls epigenetisch generierten Synapsencodes. Daraus wäre -diese hypothetische Anmerkung sei mir an dieser Stelle gestattet - zu folgern, dass das interaktive Zusammenwirken beider Codes einen Zustand herbeiführt, den man als lebenslang modulierbaren Cerebralcode bezeichnen könnte. Man schätzt, dass es im adulten menschlichen Gehirn 100 Milliarden Neurone mit jeweils durchschnittlich 5.000 Synapsen, also insgesamt 500 Billionen Synapsen (Linden 2011: 44) gibt. Entsprechend groß muss das ursprüngliche Potenzial der Bildung von Synapsencodes zu Beginn der Entwicklung eines Menschen sein. Es öffnet sich ein schier unbegrenztes Universum epigenetischer Plastizität.

Embryonale Determination und entwicklungsabhängige Prägung beruhen auf den gleichen molekularen Mechanismen. Sie realisieren die funktionelle Integration von Zellen eines Körpers einerseits sowie von Individuen eines Sozialverbands andererseits. Beides dient dem gleichen Ziel, im Rahmen von genetisch festgelegten Entwicklungsabläufen durch adaptive, epigenetische Mechanismen den Fortbestand der Art zu sichern. Allerdings drängt sich doch ein wichtiger Unterschied auf. Während in der körperlichen Entwicklung das Ergebnis im vorgegebenen Korsett des artspezifischen Bauplans als teleologischer Vorgang in weitem Umfang vorhersehbar ist, ist dies in der emotionalen und kognitiven Entwicklung eines Menschen wohl weniger der Fall. Die Vielfalt und die Kontingenz der ihn prägenden Einflüsse und Faktoren kann nur bedingt antizipiert werden. Dazu kommt, dass sich der Mensch, insbesondere in den Großstädten, eine Umwelt schafft, die sich immer mehr von der natürlichen Umwelt, die seine bisherige Evolution geprägt hat, unterscheidet. Insofern dürfte das zukünftige Schicksal der mentalen Evolution des Menschen offen sein.

\section{Literatur:}

Bock, J., Helmeke, C., Ovtscharoff jr., W., Gruß, M. \& Braun, K. (2003). Frühkindliche emotionale Erfahrungen beeinflussen die funktionelle Entwicklung des Gehirns. Neuroforum 2: 15-20. 
Bourgeois, J.-P. (2010). The neonatal synaptic big bang. In: The newborn brain: Neuroscience and clinical applications. H. Lagercrantz, M. A. Hanson, L. R. Ment, \& D. M. Peebles, Hg. (Cambridge, UK: University Press), S. 71-84.

Bourne, J. A., Warner, C. E. \& Rosa, M.G.P. (2005). Topographic and laminar maturation of striate cortex in early postnatal marmoset monkeys, as revealed by neurofilament Immunohistochemistry. Cerebral Cortex 15(6): 740-748.

Bredy, T. W., Lin, Q., Wei, W., Baker-Andresen, D. \& Mattick, J. S. (2011). MicroRNA regulation of neuronal plasticity and memory. Neurobiology of Learning and Memory 96(1): 89-94.

Doyle, M. \& Kiebler, M. A. (2011). Mechanisms of dendric mRNA transport and its role in synaptic tagging. The EMBO Journal 30(17): 3540-3552.

Frey, U. \& Morris, R. G. M.(1997). Synaptic tagging and long-term potentiation. Nature 385(6616): 533-536.

Gilbert, S. F. (2001). Continuity and change: paradigm shifts in neural induction. International Journal of Developmental Biology 45(1): 155-164.

Hadorn, E. (1955). Letalfaktoren in ihrer Bedeutung für Erbpathologie und Genphysiologie der Entwicklung. (Stuttgart: Thieme)

Kandel, E. (2007). Auf der Suche nach dem Gedächtnis. Die Entstehung einer neuen Wissenschaft des Geistes. (München: Pantheon)

Karin, M., Liu, Z. \& Zandi, E. (1997). AP-1 function and regulation. Current Opinion in Cell Biology 9(2): 240-246.

Linden, D. J. (2011). Das Gehirn. Ein Unfall der Natur und warum es dennoch funktioniert. (Reinbek b. Hamburg: Rowohlt).

Lorenz, K. (1935). Der Kumpan in der Umwelt des Vogels. In: ders. Über tierisches und menschliches Verhalten, Band 1 (München: Piper 1965). 
Lorenz, K. (1973). Die Rückseite des Spiegels. Versuch einer Naturgeschichte menschlichen Erkennens (München: Piper).

Mahler, M.S., Pine, F. \& Bergmann, A. (1997). Die psychische Geburt des Menschen (Frankfurt a. M.: Fischer).

Mangold, O. (1953). Hans Spemann. Ein Meister der Entwicklungsphysiologie, sein Leben und sein Werk (Stuttgart: Wissenschaftliche Verlagsgesellschaft).

Redondo, R. L. \& Morris, R. G. M. (2011). Making memories last: the synptic tagging and capture hypothesis. Nature Reviews Neuroscience 12(1): 17-30.

Roth, T. L. \& Sweatt, J. D. (2009). Regulation of chromatin structure in memory formation. Current Oppinion in Neurobiology 19(3): 336-342.

Roux, W. (1897). Programm und Forschungsmethoden der Entwicklungsmechanik der Organismen.( Leipzig: Engelmann).

Sadananda, M. \& Bischof, H.-J. (2004). c-Fos is induced in the hippocampus during consolidation of sexual imprinting in the zebra finch (Taeniopygia guttata). Hippocampus 14(1): 19-27.

Slack, J. M. W. (1999). Egg \& Ego. An almost true story of Life in the Biology Lab (New York, NY: Springer).

Spork, P. (2009). Der zweite Code. Epigenetik - oder Wie wir unser Erbgut steuern können. (Hamburg: Rowohlt).

Sweatt, J. D. (2009). Experience-dependent epigenetic modifications in the central nervous system. Biological Psychiatry 65(3):191-197.

Weiner, J. (2002). Zeit, Liebe, Erinnerung. Auf der Suche nach den Ursprüngen des Verhaltens. (Berlin: Berlin Taschenbuch-Verlag).

Woltereck, R. (1909). Weitere experimentelle Untersuchungen über Artveränderung, speziell über das Wesen quantitativer Artunterschiede bei Daphniden. Verhandlungen der 
Deutschen Zoologischen Gesellschaft 19: 110-173.

\section{Erschienen in/Previously published in:}

Kulturen der Epigenetik: Vererbt, codiert, übertragen. Vanessa Lux \& Jörg Thomas Richter, Hrsg. (Berlin: DeGryuter 2014). 